\title{
Strategies for intranasal delivery of vaccines
}

\author{
Mehfuz Zaman • Saranya Chandrudu • \\ Istvan Toth
}

Published online: 12 July 2012

(C) The Author(s) 2012. This article is published with open access at Springerlink.com

\begin{abstract}
The vast majority of human pathogens colonize and invade at the mucosal surfaces. Preventing infection at these sites via mucosally active vaccines is a promising and rational approach for vaccine development. However, it is only recently that the stimulation of local immunity at the mucosal surfaces has become a primary objective in addition to inducing systemic immunity. This review describes vaccine formulations designed for mucosal delivery to the nasal-associated lymphoid tissue, via intranasal administration. The association of antigens with mucosal adjuvants and delivery systems is emphasised.
\end{abstract}

Keywords Mucosal adjuvant $\cdot$ Nasal vaccine $\cdot$ Immunity · Vaccine delivery

\section{Introduction}

Vaccination is one of the most successful accomplishments of medical science. Diseases that were prevalent are now increasingly rare because of the widespread use of vaccines. Next to clean water, no single intervention has had such an overwhelming effect on reducing mortality from childhood diseases as the use of vaccines [1]. The smallpox vaccine has eradicated a disease that had a $30 \%$ fatality rate [2]. After over two decades of intensive efforts, the global polio eradication initiative is approaching its final stage aided by

M. Zaman · S. Chandrudu $\cdot$ I. Toth

School of Chemistry and Molecular Biosciences (SCMB),

The University of Queensland,

St. Lucia 4072, QLD, Australia

I. Toth $(\bowtie)$

School of Pharmacy, The University of Queensland,

Brisbane, QLD, Australia

e-mail: i.toth@uq.edu.au the two polio vaccines developed by Jonas Salk and Albert Sabin [3]. Nevertheless, next-generation vaccines are required to combat prevalent diseases.

An important field in the development of next-generation vaccines is the development of vaccines suitable for mucosal immunization. Most viral and bacterial infections start at the mucosal surfaces; thus, immunity against infective agents may depend on the induction of a mucosal immune response. As a result, for certain infectious agents, the mucosal route is the most appropriate method of immunization because it has been reported to induce both mucosal and systemic immune responses $[4,5]$.

Mucosal vaccination can be achieved via a number of routes including oral, intranasal, pulmonary, rectal, or vaginal [6]. Of these, the nasal route is the most straightforward and is suitable for vaccine administration. Advantages and disadvantages of nasal vaccination are summarized in Table 1 . The prime inductive site for mucosal immunity in the nasopharyngeal tract in rodents is the nasal-associated lymphoid tissue (NALT) [7-9]. NALT is considered the rodent equivalent of Waldeyer's ring (Fig. 1), the lymphoid tissues (tonsils) present in humans [10]. Waldeyer's ring forms a protective site at the opening of the pharynx to provide immunity [11]. Protection at this mucosal surface is correlated with secretory immunoglobulin-A (sIgA) antibodies which, alongside other innate defence mechanisms, provide additional protection from pathogens [12]. Murine NALT is a functional analogue to human tonsils, and animal research has provided insight into human immunology. Such studies are essential before any human trials of vaccine candidates. However, human immunity and biological composition can differ from those of animal models. For nasal vaccine development, development of appropriate in vitro models has provided a reproducible approach in which phenotypic and physiological features of the NALT can be simulated [13]. Techniques to collect and analyse mucosal secretions and cell types have also provided a rational approach for evaluation of vaccine efficacy [14]. 
Table 1 Advantages and disadvantages of intranasal vaccination

\begin{tabular}{ll}
\hline Advantages & Disadvantages \\
\hline Needle-free thus patient compliance & Rapid clearance \\
Non-invasive & Inefficient uptake \\
Small antigenic dose & Lack of human compatible mucosal adjuvant \\
Induction of systemic and mucosal immunity & \\
Immunity at primary and distal mucosal sites & \\
\hline
\end{tabular}

NALT comprises a organised structure of cells involved in the induction of an immune response, including dendritic cells, T cells and B cells, which are covered by an epithelial layer of cells containing distinctive cells called $\mathrm{M}$ cells [9]. $M$ cells in the NALT are the sites of antigen uptake for induction of mucosal immunity [15]. Whilst small soluble antigens are able to penetrate the nasal epithelium, particulate antigens are mainly taken up by M cells in the NALT [16]. Antigen is actively transported by $\mathrm{M}$ cells, to reach dendritic cells, macrophages and $\mathrm{B}$ cells, for processing and presentation [17]. Consequent activation of antigen-specific CD4+ T helper cells (Th cells) interact with B cells which develop into IgA committed (IgA+). IgA+ B cells move to effector sites such as the nasal passage where they differentiate into IgA-producing plasma cells and secrete $\operatorname{IgA}$ in dimers. Dimeric IgA then become S-IgA by binding to the polymeric Ig receptor which transports IgA to effector sites [18]. An overview of the immune response in NALT is summarized in Fig. 2. S-IgA are able to bind toxins, bacteria or viruses and neutralize their activity, thus preventing entry into the body or reaching the internal organs. Whilst this can prevent infection through the mucosal epithelium, certain pathogens such as group A streptococcus can concurrently infect through the skin. The advantage of vaccination at the mucosal surface by intranasal administration is the induction of mucosal and systemic immune responses, whereas traditional parenteral administration generally only results in systemic immune responses. Therefore, in the context of group A streptococcus and other pathogens which concurrently infect

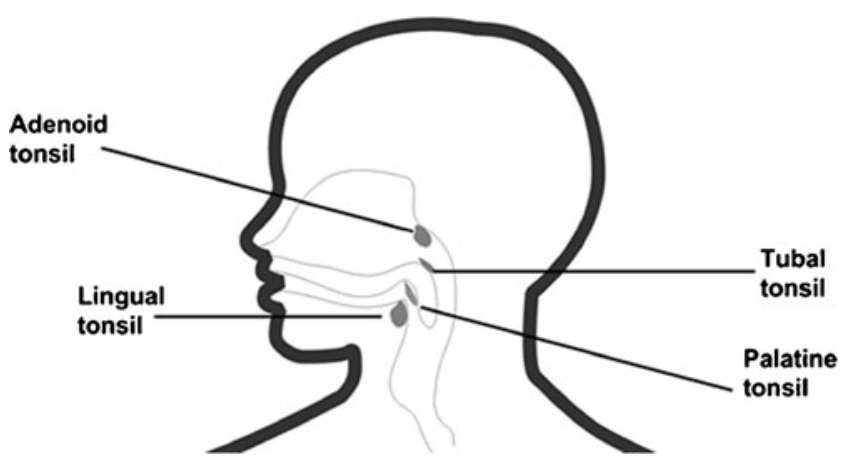

Fig. 1 Waldeyer's ring is an induction site for mucosal responses. It is formed by the lymphoid tissues near the opening of the respiratory and digestive systems. It consists of the adenoid, tubal, palatine and lingual tonsils through the mucosa and systemic sites, an ideal vaccine could be an intranasally administered vaccine eliciting neutralizing IgA preventing colonization of the throat and systemic IgG antibodies facilitating clearance from systemic sites [11].

An effective vaccine formulation for intranasal delivery maintains the antigen in a stable form, ensures the antigen, remains in the nasopharyngeal region long enough for the antigen to interact with the lymphatic system and stimulates the immune system - with or without additional adjuvants - to provide long-term immunity [19]. This review discusses the leading strategies in delivery systems and adjuvants currently under investigation for the development of nasal vaccines.

\section{Attenuated intranasal vaccines}

Nasally delivered influenza vaccines based on live-attenuated strains are currently under investigation to prevent seasonal and pandemic influenza. Live-attenuated vaccines have the advantage of mimicking natural infection by presenting influenza antigens in their native conformation to the nasal mucosal tissues, without inducing flu-like symptoms [19]. An example of this type of vaccine is FluMist by Astra-Zeneca, the first nasal trivalent vaccine for seasonal influenza [20,21]. When compared with an injectable trivalent influenza vaccine, the FluMist vaccine conferred a longer duration of protection, better cross-protection, enhanced efficacy and both mucosal and systemic immunity [19]. Despite the promise of the liveattenuated influenza vaccine strategy, adverse side effects have been reported including safety concerns for patients with allergy or asthma as well as irregular side effects similar to Bell's palsy [19].

\section{Enhancement of mucoadhesion}

Most antigens have little to no affinity for the nasal epithelium and tend to be removed quickly by mucociliary clearance [22, 23]. Extending the nasal residence time by co-administering the antigen with mucoadhesives is a logical approach to enhance absorption and residence time to facilitate interaction with the immune system [16, 24]. Significant results with biodegradable, mucoadhesive polymeric carriers have highlighted their use for mucosal vaccine delivery. Polymers such 
Fig. 2 Scheme of immunological pathway for induction of local mucosal response in NALT

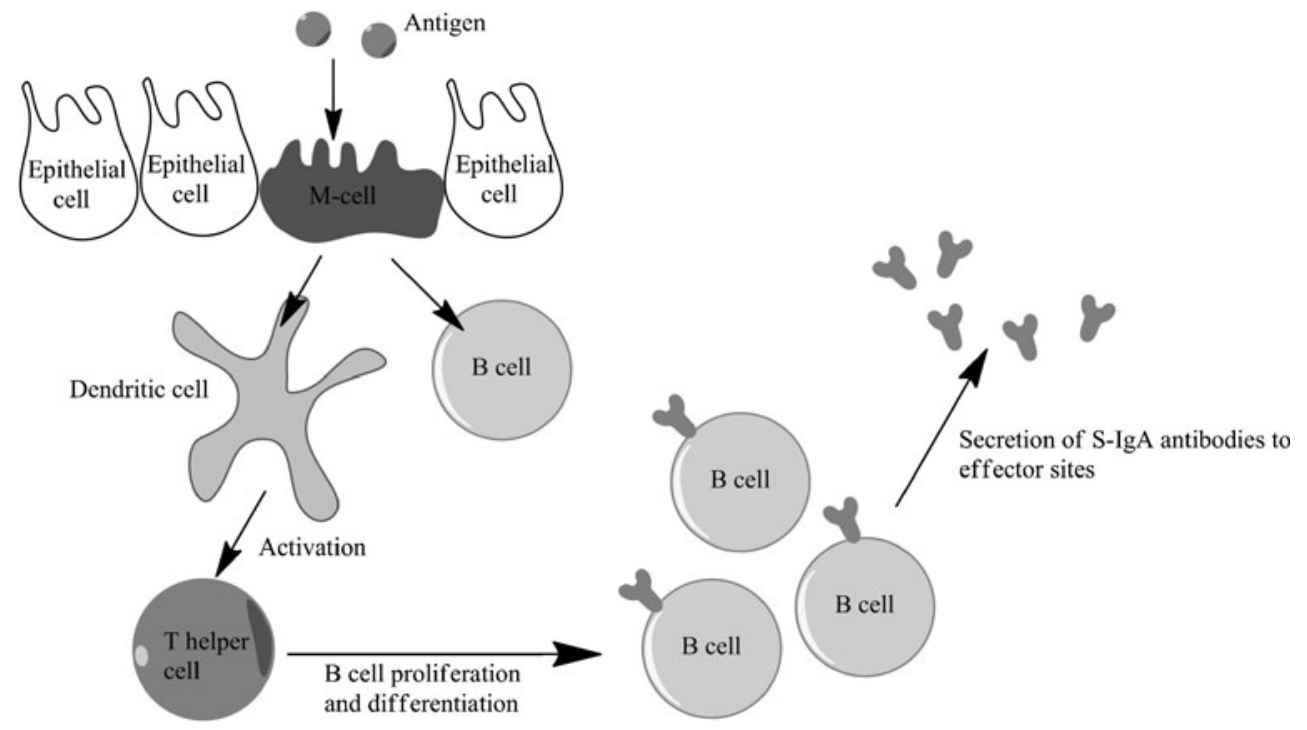

as polylactide-co-glycolide (PLGA), chitosan, alginate and carbopol have been explored for the delivery of antigens via the intranasal routes [23]. Hydrophilic polymers, like sodium alginate and carbopol, absorb to the mucus by forming hydrogen bonds, consequently enhancing nasal residence time $[25,26]$.

Sodium alginate is a linear copolymer and consists of 1 -4-linked $\beta$-D-mannuronic acid and 1-4-linked $\alpha$-L-guluronic acid residues. Sodium alginate is biocompatible and mucoadhesive and has been used for controlled delivery of drugs following intranasal administration [27, 28]. In vivo studies showed that the therapeutic efficacy of a drugsodium alginate formulation significantly improved in comparison to nasal administration of drug alone [27]. Good biocompatibility, low cost and ease of preparation are the major advantages of using alginate polymers for the delivery of an antigen [29]. Extensive use of sodium alginate as an antigen delivery system to improve the efficacy of mucosal immunization in livestock has been carried out [30]. For example, induction of systemic and mucosal immune response in cattle by intranasal administration of antigen in alginate microspheres has been reported [31].

Carbopol is a cross-linked polyacrylic acid polymer [32]. When mixtures of starch and carbopol have been used as carriers of influenza virus antigen, systemic antigen-specific IgG responses were induced after intranasal delivery [33]. The level of IgG and the immune response kinetics were improved by the presence of carbopol [33]. Carbopol has been used experimentally as a delivery system to enhance adhesion to mucosal surfaces and may facilitate enhanced protection of peptides and proteins against enzymatic degradation [34]. Carbopol has received considerable attention delivery of proteins, and Witschi and Mrsny showed (using bovine serum albumin as model antigen) that carbopol gels can be used for nasal protein delivery [35].
Chitosan is a non-toxic linear polysaccharide produced by chitin deacetylation. Chitin is a naturally occurring polymer found in the exoskeletons of insects and crustaceans [36]. As a cationic polymer, chitosan interacts with negatively charged mucin by ionic interactions [37-39]. Mucociliary clearance is decreased, and a transient effect on the paracellular transport of antigens has been observed [40]. In addition to being a mucoadhesive, chitosan has also been reported to have adjuvant properties that enhance humoral and cellular immune responses [41-43] via opening of intercellular tight junctions, which favours the transport of antigens [44]. Combination of transient opening of tight junctions and mucoadhesive properties most likely enables the interaction of the chitosan-antigen formulation to interact with the lymph nodes of the nasal cavity, leading to improved immunological responses [45]. For controlled vaccine release, chitosan nanoparticles are reported to be effectively taken up by antigen presenting cells and induce strong mucosal and systemic immune responses against antigens [36, 46, 47].

Chitosan-based formulations have been used to improve the delivery and immunogenicity of a variety of antigens. Luteinizing hormone-releasing hormone (LHRH) displays poor immunogenicity and requires the presence of an adjuvant. Chitosan-based formulations were studied as a potential adjuvant for a vaccine against LHRH. A reduction in animal steroidogenesis and spermatogenesis was observed when chitosan was used as an adjuvant, showing the ability of this delivery system to neutralize LHRH [48]. The influenza A, matrix protein 1 (M1), is highly conserved and can form the basis of a vaccine. Mice intranasally administered M1 and chitosan were challenged with lethal doses of H9N2, H1N1 and H5N1 viruses [46]. Effective crossprotection against influenza virus was observed for the vaccine candidate [35]. The results showed chitosan had 
an improvement over the efficacy of the M1-based vaccine alone [46]. Additionally, nasal immunization with $N$-trimethyl chitosan (TMC)-based nanoparticles increased the nasal residence time of ovalbumin, a model antigen. After intra-mucosal administration, slow antigen-releasing TMC nanoparticles did not induce detectable antibody titres whereas fast antigen-releasing TMC nanoparticles showed high sIgA levels and serum antibody titres [49]. It was found that these nanoparticles were mucoadhesive and stimulated the maturation of dendritic cells [49]. Furthermore, a formulation of chitosan and DNA encoding a viral protein from coxsackievirus B3 was shown to induce high levels of mucosal $\operatorname{sg} A$ and serum IgG [50]. Following intranasal challenge with lethal CVB3, significant reduction of viral load was observed [50]. Chitosan was reported to slow down the nasal mucociliary clearance and prolong the contact period between the NALT and the chitosan-antigen complex $[50,51]$. These examples with chitosan illustrate that the co-administration of antigens with mucoadhesive agents can enhance efficacy, potentially reduce antigen dose and generally facilitate the development of mucosal vaccine delivery systems [52]. Since most vaccines alone are not sufficiently taken up after mucosal administration, coadministered with penetration enhancers, adjuvants or encapsulation in particles are various approaches to overcome this [23]. Immunogenicity enhancing properties of chitosan due to combination of transient opening of tight junctions and mucoadhesive properties makes it promising for mucosal vaccine delivery.

\section{Particulate delivery systems}

Particulate delivery systems used for nasal administration of vaccines include liposomes, immune-stimulating complexes (ISCOMs) and polymeric particles-including virosomes [19, 53-55]. Particulate antigens use the transcellular route to reach the lymphoid tissues and target $\mathrm{M}$ cells. $\mathrm{M}$ cells, which are part of the NALT, act as portals of entry for antigens to enter regions containing professional antigen presenting cells (dendritic cells), B cells and T cells, thereby contributing to both humoral and cellular immune responses $[15,17,56,57]$. Particulate systems have the capacity to present multiple copies of the antigen and tend to have a similar size to pathogens, mimicking natural infection.

Liposomes are particulate vesicles composed of different ratios of lipids and cholesterol enclosing an aqueous core, enabling the incorporation of a wide variety of antigens. The immunogenicity conferred by liposomes is due to: their ability to accommodate multiple copies of antigens, preferential uptake by macrophages, protection within the biological environment and effects on the intracellular processing of antigen [19, 58]. Surface-modified (glycol chitosan or oligomannose coated) liposomes have been shown to elicit humoral and cellular immune responses that were significant (when compared to antigen alone) following intranasal administration $[59,60]$. Surface charge tends to influence the immunogenicity of mucosal liposomal vaccine formulations. Fusogenic and cationic-fusogenic liposomes encapsulated with antigens have been demonstrated to effectively stimulate a mucosal immune response [61].

ISCOMs are made up of saponin, as an adjuvant, along with lipids and an antigen, and are generally held together by hydrophobic interaction between the constituents. The essential components to form ISCOMs are cholesterol and saponin [62]. Their inherent particulate nature, multimeric antigen presentation and potent immunostimulatory activity make ISCOMs an attractive choice for vaccine delivery [19, 63-65]. Several studies have shown that ISCOMincorporated antigens induce specific local and systemic immune responses following intranasal administration, conferring protection against influenza, respiratory syncytial virus and hepatitis B [66-69].

Virosomes are an alternative particulate delivery form composed of extracted glycoproteins from virus particles and mimic viral structures. The viral glycoproteins present on virosomes have a high affinity for the mucosal surfaces of the respiratory tract [70]. Virosomes enable efficient induction of humoral and cellular responses and target dendritic cells [71-76]. They have been demonstrated to be efficient nasal delivery systems for several antigens, including DNA [75], and influenza [77, 78] and HIV proteins [79].

Particulate systems are promising for the nasal delivery of vaccines, enhancing uptake by antigen-presenting cells, conferring a depot effect and protecting the antigen from degradation. Further research in the physicochemical properties of particulates that influence immunogenicity will contribute to the development of nasal vaccine delivery systems.

\section{Lipopeptide-based delivery systems}

Lipopeptides of bacterial origin, or their synthetic derivatives, represent potent immunostimulants when given in combination with peptide or protein antigens [80]. Examples of lipid moieties include tri-palmitoyl-S-glyceryl cysteine (Pam3Cys), di-palmitoyl-S-glyceryl cysteine (Pam2Cys), single/multiple-chain palmitic acids and lipoamino acids (LAAs) (Fig. 3). The desirable immunological activity of these lipid moiety conjugates arises from their intrinsic adjuvant properties, such as B cell and macrophage activators, ability to induce maturation of dendritic cells and promote an inflammatory response [81-85]. Generally, this occurs via signalling through receptors of the immune system that recognise these lipid moieties as pathogenassociated molecular patterns [86-89]. 
Fig. 3 The structure of the lipid moieties used to enhance immunogenicity of weak antigens
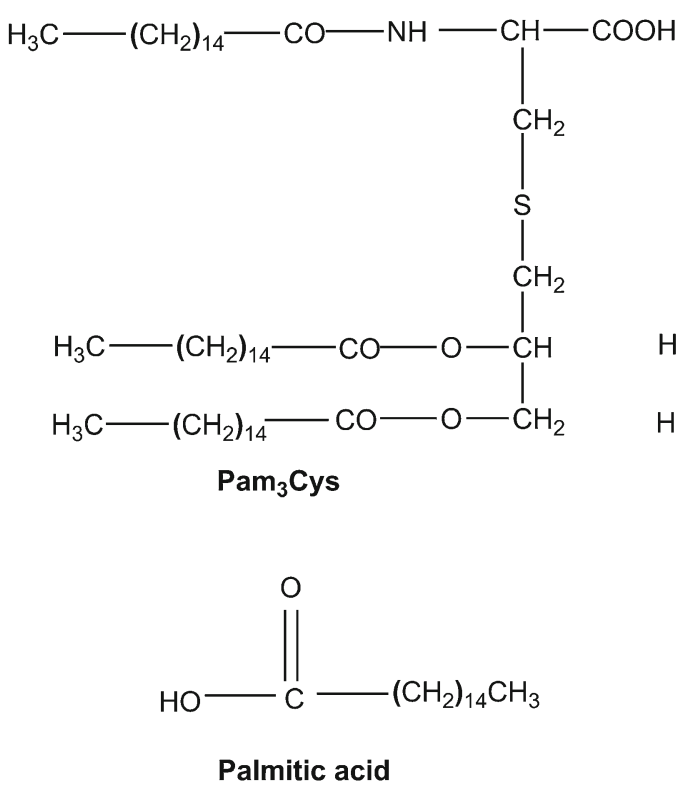
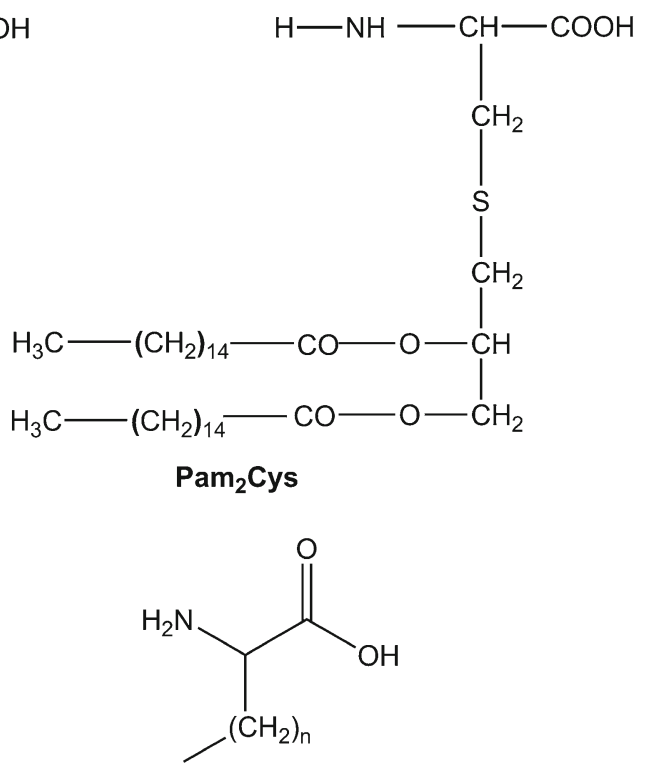

Lipoamino acid
Lipopeptides containing Pam3Cys and Pam2Cys have been evaluated for development of immunocontraceptive vaccines. When administered intranasally, the constructs were highly immunogenic, capable of inducing high titres of antibodies and efficiently sterilized female mice when administered alone in saline [90]. A Pam2Cys-based vaccine against Streptococcus pyogenes had the ability to induce mucosal and systemic antibodies following intranasal immunization and conferred protection against bacterial challenge [91]. For influenza, Pam2Cys-based lipopeptides have been shown to confer protection by inducing a long-term cellular response [92].

Lipopeptide vaccines based on a palmitic acid moiety may present an ideal strategy against pathogens that infect mucosal surfaces. Intranasal administration of a palmitoylated lipopeptide vaccine for human cytomegalovirus (a herpes virus) elicited both systemic and local mucosal cellular responses when administered intranasally [93]. Herpes simplex virus type 2 peptide epitopes conjugated to a palmitic acid moiety have shown the ability to prevent transmission and/or limit the severity of diseases [94].

A library of lipopeptide vaccine candidates composed of a S. pyogenes B cell epitope, a T helper epitope and a lipid moiety based on LAAs (Fig. 3) has also been investigated (Fig. 4). The orientation of each component of the lipopeptide was optimized to elicit a strong immune response following intranasal immunization. The antibody titres elicited in response to these lipopeptides provided important information for the design of an effective, lipopeptide-based mucosal vaccine $[95,96]$. Interestingly, the lipopeptides were self-adjuvanting, negating the use of additional adjuvants, which could easily be applied to other peptide-based vaccines. An important consideration for lipopeptide vaccines is ensuring that the lipid moiety is easy to synthesize, couple to the antigen, purify and formulate. As a result, LAAs are an attractive option because they can be synthesized and coupled to peptide antigens using standard peptide synthesis methods [97].

Lipopeptides provide several advantages for nasal vaccine development. Firstly, they negate the requirement for adjuvant, which has the potential to induce adverse side effects. Secondly, lipopeptide vaccine candidates can induce cellular and antibody responses, allowing control over the desired immune response. Current pre-clinical and clinical trials with lipopeptides suggest they are effective, non-toxic and can be synthesized using current methods of peptide synthesis to a high purity and yield. The lipopeptide-based approach for the nasal delivery of vaccines induces effective mucosal and systemic immune responses [82].

\section{DNA-based vaccines}

DNA vaccines have been investigated for intranasal mucosal vaccine development. DNA-based vaccines where antibodies raised to specific proteins involved in immunity to infection have shown promise in animal models. The composition and negative charge of DNA generally hinders the entrapment efficiency and stability of vaccine formulations. Several investigators have used cationic components such as chitosan and polyethylenimine (PEI) to complex with DNA antigens in the form of nano-/microparticles for intranasal delivery [19]. Plasmid DNA containing chitosan nanoparticles for nasal immunization against nucleocapsid (N) protein of severe acute respiratory syndrome coronavirus (SARS-CoV) showed elicitation of mucosal IgA as well as systemic IgG against $\mathrm{N}$ protein [98]. SARS DNA vaccine 


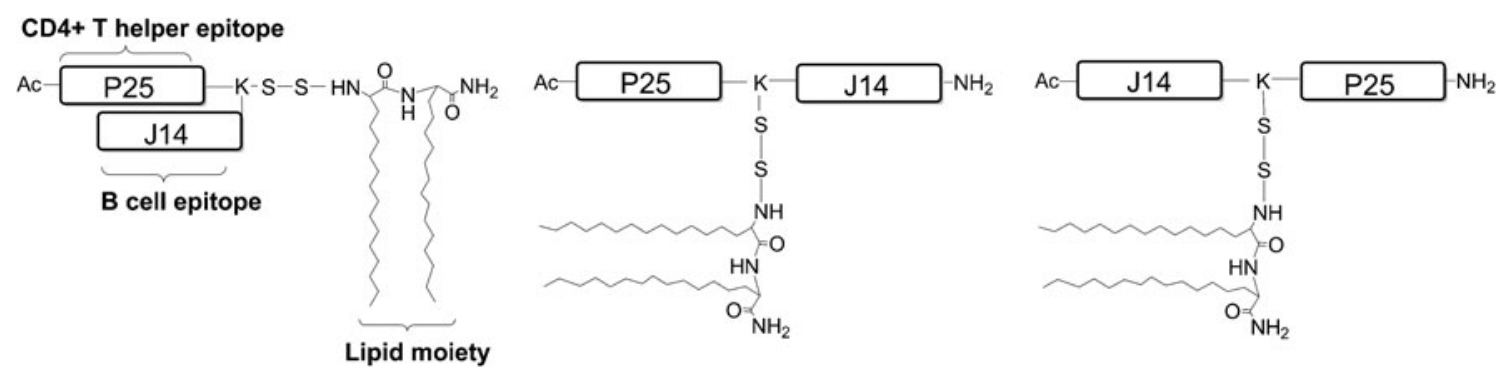

Fig. 4 Structures of lipopeptides with a T helper epitope (P25), S. pyogenes B cell epitope (J14) and a lipid moiety based on LAAs

complexed with other cationic polymers effectively delivered the plasmid DNA to induce antigen-specific humoral and cellular immune responses [99]. PEI is of particular interest for intranasal delivery due to reported many-fold increase in gene transfer in the respiratory tract. Using PEI formulation, intranasal vaccination with DNA-encoding influenza A H5N1 or H1N1 antigens, high levels of antibodies were detected in bronchoalveolar lavages and the serum [100].

Cationic PLGA particles have also been used as an intranasal delivery system for administering foot and mouth disease (FMDV) vaccine encoding the FMDV capsid protein [101]. Intranasal delivery of the cationic PLGA particles containing FMDV DNA vaccine formulations enhanced protective immunity against FMDV [101]. Whilst DNA vaccines have been shown promise for intranasal vaccine delivery, the technology requires further research to develop effective vaccines [19].

\section{Mucosal adjuvants}

Improving the immunogenicity of antigens through the use of adjuvants (which can also act as delivery systems) is a rational approach for vaccine development. Nevertheless, achieving a potent adjuvant effect whilst avoiding reactogenicity or toxicity is a major challenge. Peptide and protein antigens suffer from poor immunogenicity and require the use of adjuvants. However, existing licensed adjuvants, such as alum and to a lesser extent MF59, are not suitable as adjuvants for mucosal vaccine administration and in general do not induce mucosal antibodies [102]. Unlike the systemic immune system, mucosal surfaces regularly encounter an extensive range of foreign material. To accommodate this, the mucosal immune system must be selective in responding to antigens in order to avoid undesirable immune responses and excessive activation of the immune system [103]. Thus, induction of mucosal immunity is more difficult, and novel strategies are critical to the successful development of mucosal adjuvants.

Dendritic cells and $\mathrm{M}$ cells are the major cell types to be targeted by a mucosal adjuvant. $\mathrm{CpG}$ oligodeoxynucleotides (CpG ODN) Flt3 ligand and monophosphoryl lipid A (MLA) represent dendritic cell targeting ligands [104], whilst Flt3 is a growth factor reported to stimulate dendritic cells [105]. CpG ODN mimics the immunostimulatory effects of bacterial DNA and is known to target plasmacytoid dendritic cells for their activation, maturation [106]. Mucosal administration of CpG ODN with model antigens, including influenza virus and tetanus toxoid, effectively elicited antigen-specific immunity [107, 108].

Furthermore, Flt3 has been demonstrated as a safe adjuvant with nasal dendritic cell targeting properties that confer protection against fatal pneumococcal pneumonia in mice [109]. To this end, CpG ODN and Flt3 represent mucosal dendritic cell targeting adjuvants for the induction of antigen-specific, protective mucosal immune responses.

Bacterial lipopolysaccharide is a potent immunostimulatory agent but exhibits extreme toxicity [110]. Chemical modifications to alleviate its toxic effects resulted in the identification of MLA (Fig. 5) [111]. The clinical grade MLA formulation corresponds to the main active component of Corixa's MPL adjuvant [111]. In numerous preclinical and clinical studies, MPL has proven to be a potent yet non-toxic adjuvant. It has been used extensively as an adjuvant in human

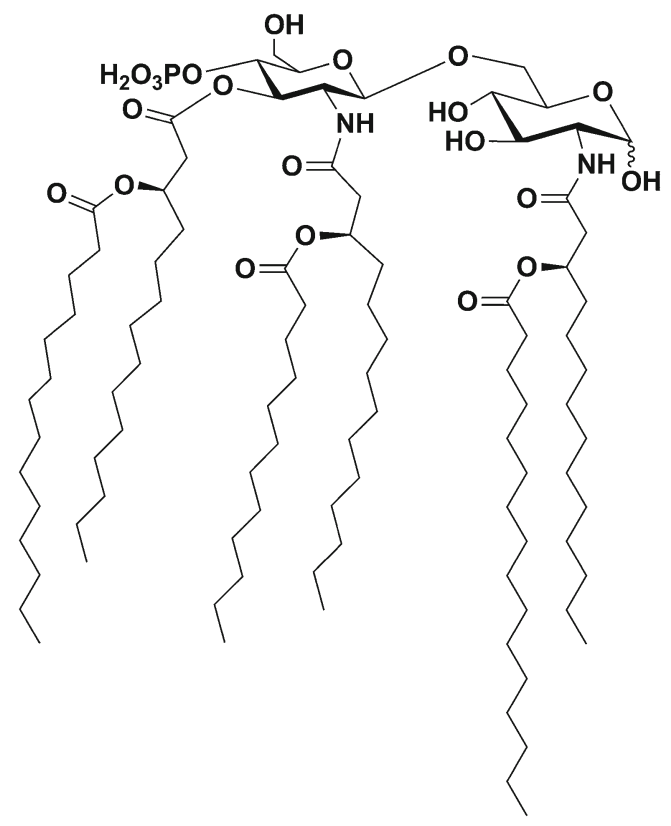

Fig. 5 Chemical structure of MLA 
vaccine trials for several infectious diseases and cancer [111]. Effectiveness of MPL as a mucosal adjuvant was investigated following intranasal administration of a formulation of MPL added to soluble antigen or liposomes encapsulating antigen [112]. The liposomal antigen formulation with MPL resulted in IgA responses that were consistently higher than seen in mice immunized with liposome antigen or free antigen without MPL [112]. These results demonstrated the effectiveness of MPL as an adjuvant for potentiating mucosal immune responses.

$M$ cells provide a portal of entry for pathogens, through which they can invade the host. Identification of the molecules that are required for bacterial or viral entry through $\mathrm{M}$ cells may provide a rational basis for developing an effective mucosal adjuvant/delivery system [104]. For example, reoviruses initially infect through $\mathrm{M}$ cells using their surface protein sigma-1 (pol) [113]. An M cell-targeting DNA vaccine formulation consisting of plasmid DNA and the reovirus p 1 induced significant mucosal and systemic immunity [114]. This suggests that $\mathrm{M}$ cell targeting could be a useful approach for the development of a mucosal vaccine.

The above examples represent a sample of the strategies that could be used to facilitate the development of novel mucosal adjuvants. However, mucosal adjuvants must still overcome the two major hurdles: effectiveness and safety. These are both relatively more difficult objectives compared with the development of vaccines that elicit systemic immunity, due to the uniqueness and complexity of the mucosal immune system.

\section{Conclusion}

The development of novel, mucosally active, intranasally administered vaccines has the potential to provide immunity against a myriad of infectious diseases. Mucosal administration of vaccines presents an ideal strategy against many pathogens that infect via mucosal surfaces. A suitable combination of adjuvants targeting $\mathrm{M}$ cells or dendritic cells and delivery systems that are mucoadhesive could facilitate the development of effective nasal vaccines. The use of the non-invasive, needle-free nasal route is advantageous for vaccination programmes, since it can enhance patient compliance and reduce need to be administered by specialised healthcare workers.

Open Access This article is distributed under the terms of the Creative Commons Attribution License which permits any use, distribution, and reproduction in any medium, provided the original author(s) and the source are credited.

\section{References}

1. Ricciardi W. The old Edward Jenner and the new public health: the future of vaccines in Europe. Eur J Public Health. 2008;18 (4):353.
2. Ellner PD. Smallpox: gone but not forgotten. Infection. 1998;26 (5):263-9.

3. Obregon R, Chitnis K, Morry C, Feek W, Bates J, Galway M, et al. Achieving polio eradication: a review of health communication evidence and lessons learned in India and Pakistan. Bull World Health Organ. 2009;87(8):624-30.

4. Almeida AJ, Alpar HO. Nasal delivery of vaccines. J Drug Target. 1996;3(6):455-67.

5. Boyaka PN, Tafaro A, Fischer R, Leppla SH, Fujihashi K, McGhee JR. Effective mucosal immunity to anthrax: neutralizing antibodies and Th cell responses following nasal immunization with protective antigen. J Immunol. 2003;170(11):5636-43.

6. Neutra MR, Kozlowski PA. Mucosal vaccines: the promise and the challenge. Nat Rev Immunol. 2006;6(2):148-58.

7. Zuercher AW, Coffin SE, Thurnheer MC, Fundova P, Cebra JJ. Nasal-associated lymphoid tissue is a mucosal inductive site for virus-specific humoral and cellular immune responses. J Immunol. 2002;168(4):1796-803.

8. Hiroi $\mathrm{T}$, Iwatani $\mathrm{K}$, Iijima $\mathrm{H}$, Kodama $\mathrm{S}$, Yanagita $\mathrm{M}$, Kiyono H. Nasal immune system: distinctive Th0 and Th1/ Th2 type environments in murine nasal-associated lymphoid tissues and nasal passage, respectively. Eur J Immunol. 1998;28(10):3346-53.

9. Wu HY, Nguyen HH, Russell MW. Nasal lymphoid tissue (NALT) as a mucosal immune inductive site. Scand J Immunol. 1997;46(5):506-13.

10. Cesta MF. Normal structure, function, and histology of mucosaassociated lymphoid tissue. Toxicol Pathol. 2006;34(5):599-608.

11. Brandtzaeg P. Immune functions of nasopharyngeal lymphoid tissue. Adv Otorhinolaryngol. 2011;72:20-4.

12. Whaley KJ, Zeitlin L. Preventing transmission: plant-derived microbicides and mucosal vaccines for reproductive health. Vaccine. 2005;23(15):1819-22.

13. Corr SC, Gahan CC, Hill C. M-cells: origin, morphology and role in mucosal immunity and microbial pathogenesis. FEMS Immunol Med Microbiol. 2008;52(1):2-12.

14. Guy B. Evaluation of events occurring at mucosal surfaces: techniques used to collect and analyze mucosal secretions and cells. Clin Diagn Lab Immunol. 2002;9(4):753-62.

15. Fujimura Y. Evidence of $M$ cells as portals of entry for antigens in the nasopharyngeal lymphoid tissue of humans. Virchows Arch. 2000;436(6):560-6.

16. Davis SS. Nasal vaccines. Adv Drug Deliv Rev. 2001;51(1-3):21-42.

17. Ogasawara N, Kojima T, Go M, Takano K, Kamekura R, Ohkuni $\mathrm{T}$, et al. Epithelial barrier and antigen uptake in lymphoepithelium of human adenoids. Acta Otolaryngol. 2011;131(2):116-23.

18. Johansen FE, Kaetzel CS. Regulation of the polymeric immunoglobulin receptor and IgA transport: new advances in environmental factors that stimulate $\mathrm{pIgR}$ expression and its role in mucosal immunity. Mucosal Immunol. 2011;4(6):598-602.

19. Jabbal-Gill I. Nasal vaccine innovation. J Drug Target. 2010;18 (10):771-86.

20. Belshe RB, Mendelman PM, Treanor J, King J, Gruber WC, Piedra $\mathrm{P}$, et al. The efficacy of live attenuated, cold-adapted, trivalent, intranasal influenzavirus vaccine in children. N Engl J Med. 1998;338(20):1405-12.

21. Izurieta HS, Haber P, Wise RP, Iskander J, Pratt D, Mink C, et al. Adverse events reported following live, cold-adapted, intranasal influenza vaccine. JAMA. 2005;294(21):2720-5.

22. Velasquez LS, Shira S, Berta AN, Kilbourne J, Medi BM, Tizard I, et al. Intranasal delivery of Norwalk virus-like particles formulated in an in situ gelling, dry powder vaccine. Vaccine. 2011;29 (32):5221-31.

23. Garg NK, Mangal S, Khambete H, Tyagi RK. Mucosal delivery of vaccines: role of mucoadhesive/biodegradable polymers. Recent Pat Drug Deliv Formul. 2010;4(2):114-28. 
24. Illum L. Nasal drug delivery-possibilities, problems and solutions. J Control Release. 2003;87(1-3):187-98.

25. Yang J, Zhao J, Fang Y. Calorimetric studies of the interaction between sodium alginate and sodium dodecyl sulfate in dilute solutions at different $\mathrm{pH}$ values. Carbohydr Res. 2008;343 (4):719-25.

26. Sahoo S, Chakraborti CK, Mishra SC. Qualitative analysis of controlled release ciprofloxacin/carbopol 934 mucoadhesive suspension. J Adv Pharm Technol Res. 2011;2(3):195-204.

27. Rajinikanth PS, Sankar C, Mishra B. Sodium alginate microspheres of metoprolol tartrate for intranasal systemic delivery: development and evaluation. Drug Deliv. 2003;10(1):21-8.

28. Bertram U, Bodmeier R. In situ gelling, bioadhesive nasal inserts for extended drug delivery: in vitro characterization of a new nasal dosage form. Eur J Pharm Sci. 2006;27(1):62-71.

29. Singh M, O'Hagan D. The preparation and characterization of polymeric antigen delivery systems for oral administration. Adv Drug Deliv Rev. 1998;34(2-3):285-304.

30. Sedgmen BJ, Meeusen EN, Lofthouse SA. Alternative routes of mucosal immunization in large animals. Immunol Cell Biol. 2004;82(1):10-6.

31. Rebelatto MC, Guimond P, Bowersock TL, HogenEsch H. Induction of systemic and mucosal immune response in cattle by intranasal administration of pig serum albumin in alginate microparticles. Vet Immunol Immunopathol. 2001;83(1-2):93-105.

32. Makhlof A, Fujimoto S, Tozuka Y, Takeuchi H. In vitro and in vivo evaluation of WGA-carbopol modified liposomes as carriers for oral peptide delivery. Eur J Pharm Biopharm. 2011;77(2):216-24.

33. Coucke D, Schotsaert M, Libert C, Pringels E, Vervaet C, Foreman $\mathrm{P}$, et al. Spray-dried powders of starch and crosslinked poly(acrylic acid) as carriers for nasal delivery of inactivated influenza vaccine. Vaccine. 2009;27(8):1279-86.

34. Alpar HO, Somavarapu S, Atuah KN, Bramwell VW. Biodegradable mucoadhesive particulates for nasal and pulmonary antigen and DNA delivery. Adv Drug Deliv Rev. 2005;57(3):411-30.

35. Witschi C, Mrsny RJ. In vitro evaluation of microparticles and polymer gels for use as nasal platforms for protein delivery. Pharm Res. 1999;16(3):382-90.

36. Wen ZS, Xu YL, Zou XT, Xu ZR. Chitosan nanoparticles act as an adjuvant to promote both Th1 and Th2 immune responses induced by ovalbumin in mice. Mar Drugs. 2011;9(6):1038-55.

37. Rossi S, Ferrari F, Bonferoni MC, Caramella C. Characterization of chitosan hydrochloride-mucin rheological interaction: influence of polymer concentration and polymer:mucin weight ratio. Eur J Pharm Sci. 2001;12(4):479-85.

38. Ferrari F, Rossi S, Bonferoni MC, Caramella C, Karlsen J. Characterization of rheological and mucoadhesive properties of three grades of chitosan hydrochloride. Farmaco. 1997;52(6-7):493-7.

39. Harding SE. Mucoadhesive interactions. Biochem Soc Trans. 2003;31(Pt 5):1036-41.

40. Fujimura Y, Akisada T, Harada T, Haruma K. Uptake of microparticles into the epithelium of human nasopharyngeal lymphoid tissue. Med Mol Morphol. 2006;39(4):181-6.

41. Rauw F, Gardin Y, Palya V, Anbari S, Gonze M, Lemaire S, et al. The positive adjuvant effect of chitosan on antigen-specific cellmediated immunity after chickens vaccination with live Newcastle disease vaccine. Vet Immunol Immunopathol. 2010;134 (3-4):249-58.

42. Ghendon Y, Markushin S, Krivtsov G, Akopova I. Chitosan as an adjuvant for parenterally administered inactivated influenza vaccines. Arch Virol. 2008;153(5):831-7.

43. Verheul RJ, Hagenaars N, van Es T, van Gaal EV, de Jong PH, Bruijns S, et al. A step-by-step approach to study the influence of $\mathrm{N}$-acetylation on the adjuvanticity of N, N, N-trimethyl chitosan (TMC) in an intranasal nanoparticulate influenza virus vaccine. Eur J Pharm Sci. 2012;45(4):467-74.
44. Ranaldi G, Marigliano I, Vespignani I, Perozzi G, Sambuy Y. The effect of chitosan and other polycations on tight junction permeability in the human intestinal Caco-2 cell line(1). J Nutr Biochem. 2002;13 (3):157-67.

45. Svindland SC, Jul-Larsen A, Pathirana R, Andersen S, Madhun A, Montomoli E, et al. The mucosal and systemic immune responses elicited by a chitosan-adjuvanted intranasal influenza H5N1 vaccine. Influenza Other Respi Viruses. 2012;6(2):90-100.

46. Sui Z, Chen Q, Fang F, Zheng M, Chen Z. Cross-protection against influenza virus infection by intranasal administration of M1-based vaccine with chitosan as an adjuvant. Vaccine. 2010;28 (48):7690-8.

47. McNeela EA, O'Connor D, Jabbal-Gill I, Illum L, Davis SS, Pizza M, et al. A mucosal vaccine against diphtheria: formulation of cross reacting material (CRM(197)) of diphtheria toxin with chitosan enhances local and systemic antibody and $\mathrm{Th} 2$ responses following nasal delivery. Vaccine. 2000;19(9-10):1188-98.

48. Saenz L, Neira-Carrillo A, Paredes R, Cortes M, Bucarey S, Arias JL. Chitosan formulations improve the immunogenicity of a GnRH-I peptide-based vaccine. Int J Pharm. 2009;369 (1-2):64-71.

49. Slutter B, Bal S, Keijzer C, Mallants R, Hagenaars N, Que I, et al. Nasal vaccination with $\mathrm{N}$-trimethyl chitosan and PLGA based nanoparticles: nanoparticle characteristics determine quality and strength of the antibody response in mice against the encapsulated antigen. Vaccine. 2010;28(38):6282-91.

50. Xu W, Shen Y, Jiang Z, Wang Y, Chu Y, Xiong S. Intranasal delivery of chitosan-DNA vaccine generates mucosal SIgA and anti-CVB3 protection. Vaccine. 2004;22(27-28):3603-12.

51. Aspden TJ, Mason JD, Jones NS, Lowe J, Skaugrud O, Illum L. Chitosan as a nasal delivery system: the effect of chitosan solutions on in vitro and in vivo mucociliary transport rates in human turbinates and volunteers. J Pharm Sci. 1997;86(4):509-13.

52. Illum L, Jabbal-Gill I, Hinchcliffe M, Fisher AN, Davis SS. Chitosan as a novel nasal delivery system for vaccines. Adv Drug Deliv Rev. 2001;51(1-3):81-96.

53. Sharma S, Mukkur TK, Benson HA, Chen Y. Pharmaceutical aspects of intranasal delivery of vaccines using particulate systems. J Pharm Sci. 2009;98(3):812-43.

54. Heurtault B, Frisch B, Pons F. Liposomes as delivery systems for nasal vaccination: strategies and outcomes. Expert Opin Drug Deliv. 2010;7(7):829-44.

55. Almeida AJ, Souto E. Solid lipid nanoparticles as a drug delivery system for peptides and proteins. Adv Drug Deliv Rev. 2007;59 (6):478-90.

56. Takata S, Ohtani O, Watanabe Y. Lectin binding patterns in rat nasal-associated lymphoid tissue (NALT) and the influence of various types of lectin on particle uptake in NALT. Arch Histol Cytol. 2000;63(4):305-12.

57. Zho F, Neutra MR. Antigen delivery to mucosa-associated lymphoid tissues using liposomes as a carrier. Biosci Rep. 2002;22 (2):355-69.

58. Henriksen-Lacey M, Korsholm KS, Andersen P, Perrie Y, Christensen D. Liposomal vaccine delivery systems. Expert Opin Drug Deliv. 2011;8(4):505-19.

59. Khatri K, Goyal AK, Gupta PN, Mishra N, Mehta A, Vyas SP. Surface modified liposomes for nasal delivery of DNA vaccine. Vaccine. 2008;26(18):2225-33.

60. Ishii M, Kojima N. Mucosal adjuvant activity of oligomannosecoated liposomes for nasal immunization. Glycoconj J. 2010;27 (1):115-23.

61. Tafaghodi M, Jaafari MR, Tabassi SA. Nasal immunization studies by cationic, fusogenic and cationic-fusogenic liposomes encapsulated with tetanus toxoid. Curr Drug Deliv. 2008;5(2):108-13.

62. Pearse MJ, Drane D. ISCOMATRIX adjuvant for antigen delivery. Adv Drug Deliv Rev. 2005;57(3):465-74. 
63. Duewell P, Kisser U, Heckelsmiller K, Hoves S, Stoitzner P, Koernig S, et al. ISCOMATRIX adjuvant combines immune activation with antigen delivery to dendritic cells in vivo leading to effective cross-priming of CD8+ T cells. J Immunol. 2011;187 (1):55-63.

64. Schnurr M, Orban M, Robson NC, Shin A, Braley H, Airey D, et al. ISCOMATRIX adjuvant induces efficient cross-presentation of tumor antigen by dendritic cells via rapid cytosolic antigen delivery and processing via tripeptidyl peptidase II. J Immunol. 2009;182(3):1253-9.

65. Drane D, Gittleson C, Boyle J, Maraskovsky E. ISCOMATRIX adjuvant for prophylactic and therapeutic vaccines. Expert Rev Vaccines. 2007;6(5):761-72.

66. Coulter A, Harris R, Davis R, Drane D, Cox J, Ryan D, et al. Intranasal vaccination with ISCOMATRIX adjuvanted influenza vaccine. Vaccine. 2003;21(9-10):946-9.

67. Chen M, Hu KF, Rozell B, Orvell C, Morein B, Liljestrom P. Vaccination with recombinant alphavirus or immune-stimulating complex antigen against respiratory syncytial virus. J Immunol. 2002;169(6):3208-16.

68. Kodama S, Hirano T, Noda K, Umemoto S, Suzuki M. Nasal immunization with plasmid DNA encoding P6 protein and immunostimulatory complexes elicits nontypeable Haemophilus influenzae-specific long-term mucosal immune responses in the nasopharynx. Vaccine. 2011;29(10):1881-90.

69. Pandey RS, Dixit VK. Evaluation of ISCOM vaccines for mucosal immunization against hepatitis B. J Drug Target. 2010;18 (4):282-91.

70. Walker RI. New strategies for using mucosal vaccination to achieve more effective immunization. Vaccine. 1994;12(5):387400.

71. Kammer AR, Amacker M, Rasi S, Westerfeld N, Gremion C, Neuhaus D, et al. A new and versatile virosomal antigen delivery system to induce cellular and humoral immune responses. Vaccine. 2007;25(41):7065-74.

72. Bungener L, Huckriede A, Wilschut J, Daemen T. Delivery of protein antigens to the immune system by fusion-active virosomes: a comparison with liposomes and ISCOMs. Biosci Rep. 2002;22(2):323-38.

73. Cusi MG, Zurbriggen R, Correale P, Valassina M, Terrosi C, Pergola L, et al. Influenza virosomes are an efficient delivery system for respiratory syncytial virus-F antigen inducing humoral and cell-mediated immunity. Vaccine. 2002;20(29-30):3436-42.

74. Cech PG, Aebi T, Abdallah MS, Mpina M, Machunda EB, Westerfeld N, et al. Virosome-formulated Plasmodium falciparum AMA-1 \& CSP derived peptides as malaria vaccine: randomized phase $1 \mathrm{~b}$ trial in semi-immune adults \& children. PLoS One. 2011;6(7):e22273.

75. Cusi MG, Terrosi C, Savellini GG, Di Genova G, Zurbriggen R, Correale P. Efficient delivery of DNA to dendritic cells mediated by influenza virosomes. Vaccine. 2004;22(5-6):735-9.

76. Angel J, Chaperot L, Molens JP, Mezin P, Amacker M, Zurbriggen $\mathrm{R}$, et al. Virosome-mediated delivery of tumor antigen to plasmacytoid dendritic cells. Vaccine. 2007;25(19):3913-21.

77. Gluck U, Gebbers JO, Gluck R. Phase 1 evaluation of intranasal virosomal influenza vaccine with and without Escherichia coli heat-labile toxin in adult volunteers. J Virol. 1999;73(9):7780-6.

78. Gluck R, Mischler R, Durrer P, Furer E, Lang AB, Herzog C, et al. Safety and immunogenicity of intranasally administered inactivated trivalent virosome-formulated influenza vaccine containing Escherichia coli heat-labile toxin as a mucosal adjuvant. J Infect Dis. 2000;181(3):1129-32.

79. Bomsel M, Tudor D, Drillet AS, Alfsen A, Ganor Y, Roger MG, et al. Immunization with HIV-1 gp41 subunit virosomes induces mucosal antibodies protecting nonhuman primates against vaginal SHIV challenges. Immunity. 2011;34(2):269-80.
80. Bessler WG, Heinevetter L, Wiesmuller KH, Jung G, Baier W, Huber $\mathrm{M}$, et al. Bacterial cell wall components as immunomodulators- $\mathrm{I}$. Lipopeptides as adjuvants for parenteral and oral immunization. Int $\mathrm{J}$ Immunopharmacol. 1997;19(9-10):547-50.

81. Bessler WG, Jung G. Synthetic lipopeptides as novel adjuvants. Res Immunol. 1992;143(5):548-53. discussion 79-80.

82. Bessler WG, Baier W, vd Esche U, Hoffmann P, Heinevetter L, Wiesmuller $\mathrm{KH}$ et al. Bacterial lipopeptides constitute efficient novel immunogens and adjuvants in parenteral and oral immunization. Behring Inst Mitt. 1997(98):390-9.

83. Muller MR, Wiesmuller KH, Jung G, Loop T, Humar M, Pfannes $\mathrm{SD}$, et al. Lipopeptide adjuvants: monitoring and comparison of P3CSK4- and LPS-induced gene transcription. Int Immunopharmacol. 2002;2(8):1065-77.

84. Kargakis M, Zevgiti S, Krikorian D, Sakarellos-Daitsiotis M, Sakarellos C, Panou-Pomonis E. A palmitoyl-tailed sequential oligopeptide carrier for engineering immunogenic conjugates. Vaccine. 2007;25(37-38):6708-12.

85. Yong M, Mitchell D, Caudron A, Toth I, Olive C. Expression of maturation markers on murine dendritic cells in response to group A streptococcal lipopeptide vaccines. Vaccine. 2009;27(2526):3313-8.

86. Barrenschee M, Lex D, Uhlig S. Effects of the TLR2 agonists MALP-2 and Pam3Cys in isolated mouse lungs. PLoS One. 2010;5(11):e13889.

87. Zeng W, Eriksson E, Chua B, Grollo L, Jackson DC. Structural requirement for the agonist activity of the TLR2 ligand Pam2Cys. Amino Acids. 2010;39(2):471-80.

88. Zhu X, Ramos TV, Gras-Masse H, Kaplan BE, BenMohamed L. Lipopeptide epitopes extended by an Nepsilon-palmitoyl-lysine moiety increase uptake and maturation of dendritic cells through a Toll-like receptor-2 pathway and trigger a Th1-dependent protective immunity. Eur J Immunol. 2004;34(11):3102-14.

89. Zaman M, Abdel-Aal AB, Phillipps KS, Fujita Y, Good MF, Toth I. Structure-activity relationship of lipopeptide group A streptococcus (GAS) vaccine candidates on Toll-like receptor 2. Vaccine. 2010;28(10):2243-8.

90. Zeng W, Ghosh S, Lau YF, Brown LE, Jackson DC. Highly immunogenic and totally synthetic lipopeptides as selfadjuvanting immunocontraceptive vaccines. J Immunol. 2002;169 (9):4905-12.

91. Batzloff MR, Hartas J, Zeng W, Jackson DC, Good MF. Intranasal vaccination with a lipopeptide containing a conformationally constrained conserved minimal peptide, a universal $\mathrm{T}$ cell epitope, and a self-adjuvanting lipid protects mice from group A streptococcus challenge and reduces throat colonization. J Infect Dis. 2006;194(3):325-30.

92. Deliyannis G, Kedzierska K, Lau YF, Zeng W, Turner SJ, Jackson $\mathrm{DC}$, et al. Intranasal lipopeptide primes lung-resident memory CD8 + T cells for long-term pulmonary protection against influenza. Eur J Immunol. 2006;36(3):770-8.

93. BenMohamed L, Krishnan R, Auge C, Primus JF, Diamond DJ. Intranasal administration of a synthetic lipopeptide without adjuvant induces systemic immune responses. Immunology. 2002;106 (1):113-21.

94. Zhang X, Chentoufi AA, Dasgupta G, Nesburn AB, Wu M, Zhu $X$, et al. A genital tract peptide epitope vaccine targeting TLR-2 efficiently induces local and systemic CD8+ T cells and protects against herpes simplex virus type 2 challenge. Mucosal Immunol. 2009;2(2):129-43.

95. Zaman M, Abdel-Aal AB, Fujita Y, Phillipps KS, Batzloff MR, Good MF, et al. Immunological evaluation of lipopeptide group A streptococcus (GAS) vaccine: structure-activity relationship. PLoS One. 2012;7(1):e30146.

96. Abdel-Aal AB, Zaman M, Fujita Y, Batzloff MR, Good MF, Toth I. Design of three-component vaccines against group A 
streptococcal infections: importance of spatial arrangement of vaccine components. J Med Chem. 2010;53(22):8041-6.

97. Abdel-Aal AB, Al-Isae K, Zaman M, Toth I. Simple synthetic tolllike receptor 2 ligands. Bioorg Med Chem Lett. 2011;21(19):5863-5.

98. Raghuwanshi D, Mishra V, Das D, Kaur K, Suresh MR. Dendritic cell targeted chitosan nanoparticles for nasal DNA immunization against SARS CoV nucleocapsid protein. Mol Pharm. 2012;9 (4):946-56.

99. Shim BS, Park SM, Quan JS, Jere D, Chu H, Song MK, et al. Intranasal immunization with plasmid DNA encoding spike protein of SARS-coronavirus/polyethylenimine nanoparticles elicits antigen-specific humoral and cellular immune responses. BMC Immunol. 2010;11:65.

100. Torrieri-Dramard L, Lambrecht B, Ferreira HL, Van den Berg T, Klatzmann D, Bellier B. Intranasal DNA vaccination induces potent mucosal and systemic immune responses and crossprotective immunity against influenza viruses. Mol Ther. 2011;19(3):602-11.

101. Wang G, Pan L, Zhang Y, Wang Y, Zhang Z, Lu J, et al. Intranasal delivery of cationic PLGA nano/microparticles-loaded FMDV DNA vaccine encoding IL-6 elicited protective immunity against FMDV challenge. PLoS One. 2011;6(11):e27605.

102. Moschos SA, Bramwell VW, Somavarapu S, Alpar HO. Adjuvant synergy: the effects of nasal coadministration of adjuvants. Immunol Cell Biol. 2004;82(6):628-37.

103. Holmgren J, Czerkinsky C. Mucosal immunity and vaccines. Nat Med. 2005;11(4 Suppl):S45-53.

104. Fujkuyama Y, Tokuhara D, Kataoka K, Gilbert RS, McGhee JR, Yuki Y, et al. Novel vaccine development strategies for inducing mucosal immunity. Expert Rev Vaccines. 2012;11(3):367-79.

105. Disis ML, Rinn K, Knutson KL, Davis D, Caron D, dela Rosa C, et al. Flt3 ligand as a vaccine adjuvant in association with HER-2/ neu peptide-based vaccines in patients with HER-2/neu-overexpressing cancers. Blood. 2002;99(8):2845-50.
106. Nierkens S, den Brok MH, Garcia Z, Togher S, Wagenaars J, Wassink $\mathrm{M}$, et al. Immune adjuvant efficacy of $\mathrm{CpG}$ oligonucleotide in cancer treatment is founded specifically upon TLR9 function in plasmacytoid dendritic cells. Cancer Res. 2011;71(20):6428-37.

107. Moldoveanu Z, Love-Homan L, Huang WQ, Krieg AM. CpG DNA, a novel immune enhancer for systemic and mucosal immunization with influenza virus. Vaccine. 1998;16(11-12):1216-24.

108. McCluskie MJ, Weeratna RD, Krieg AM, Davis HL. CpG DNA is an effective oral adjuvant to protein antigens in mice. Vaccine. 2000;19(7-8):950-7.

109. Kataoka K, Fujihashi K, Oma K, Fukuyama Y, Hollingshead SK, Sekine $\mathrm{S}$, et al. The nasal dendritic cell-targeting Flt3 ligand as a safe adjuvant elicits effective protection against fatal pneumococcal pneumonia. Infect Immun. 2011;79(7):2819-28.

110. Kim KH, Kim DI, Kim SH, Jung EM, Kang JH, Jeung EB, et al. Trans-10, cis-12-conjugated linoleic acid attenuates tumor necrosis factor-alpha production by lipopolysaccharide-stimulated porcine peripheral blood mononuclear cells through induction of interleukin-10. Cytokine. 2011;56(2):224-30.

111. Persing DH, Coler RN, Lacy MJ, Johnson DA, Baldridge JR, Hershberg RM, et al. Taking toll: lipid A mimetics as adjuvants and immunomodulators. Trends Microbiol. 2002;10(10 Suppl):S32-7.

112. Childers NK, Miller KL, Tong G, Llarena JC, Greenway T, Ulrich JT, et al. Adjuvant activity of monophosphoryl lipid A for nasal and oral immunization with soluble or liposome-associated antigen. Infect Immun. 2000;68(10):5509-16.

113. Helander A, Silvey KJ, Mantis NJ, Hutchings AB, Chandran K, Lucas WT, et al. The viral sigmal protein and glycoconjugates containing alpha2-3-linked sialic acid are involved in type 1 reovirus adherence to $\mathrm{M}$ cell apical surfaces. J Virol. 2003;77 (14):7964-77.

114. Wu Y, Wang X, Csencsits KL, Haddad A, Walters N, Pascual DW. $M$ cell-targeted DNA vaccination. Proc Natl Acad Sci U S A. 2001;98(16):9318-23. 